\title{
Piano training in youths with hand motor impairments after damage to the developing brain
}

This article was published in the following Dove Press journal:

Neuropsychiatric Disease and Treatment

3 August 2015

Number of times this article has been viewed

\section{Renée Lampe ${ }^{1, *}$ \\ Anna Thienel ${ }^{2}$ \\ Jürgen Mitternacht' \\ Tobias Blumenstein' \\ Varvara Turova' \\ Ana Alves-Pintol,*}

'Research Unit for Paediatric Neuroorthopaedics and Cerebral Palsy, Orthopaedics Department, Klinikum Rechts der Isar, Technische Universität München, ${ }^{2}$ Department Sonderpädagogik, Ludwig MaximiliansUniversität München, Munich, Germany

*These authors contributed equally to this work
Correspondence: Renée Lampe Markus Würth Stiftungsprofessur für Cerebralparesen und Kinderneuroorthopädie Forschungseinheit Buhl-StromaierStiftung, Orthopaedics Department, Klinikum Rechts der Isar, Technische Universität München, Ismaninger Strasse 22, 81675 Munich, Germany

Tel +498941406360

Fax +49894I40 4849

Email renee.lampe@tum.de
Abstract: Damage to the developing brain may lead to impairment of the hand motor function and negatively impact on patients' quality of life. Development of manual dexterity and finger and hand motor function may be promoted by learning to play the piano. The latter brings together music with the intensive training of hand coordination and fine finger mobility. We investigated if learning to play the piano helped to improve hand motor skills in 18 youths with hand motor disorders resulting from damage during early brain development. Participants trained 35-40 minutes twice a week for 18 months with a professional piano teacher. With the use of a Musical Instrument Digital Interface piano, the uniformity of finger strokes could be objectively assessed from the timing of keystrokes. The analysis showed a significant improvement in the uniformity of keystrokes during the training. Furthermore, the youths showed strong motivation and engagement during the study. This is nevertheless an open study, and further studies remain needed to exclude effects of growth and concomitant therapies on the improvements observed and clarify which patients will more likely benefit from learning to play the piano.

Keywords: manual skill, cerebral palsy, neurodevelopmental disorder, music, rehabilitation

\section{Introduction}

Infantile cerebral palsy (CP) is the most frequent cause of physical impairments and disability, with an incidence of two to three in 1,000 live births. ${ }^{1}$ Intrauterine infections and low birth weight are some of the risk factors associated with early brain damage. When the area of the first motor neuron is affected, impaired motor skills, increased muscle spasticity, and increased reflexes arise. ${ }^{2}$ In particular, damage to the corticospinal tract, which enervates hand motor neurons, ${ }^{3}$ leads to impairment of the hand motor function. Depending on the stage of neuronal development at the time of injury, a reorganization of the neuronal tracts can take place and partially compensate for the diminished motor function. ${ }^{4,5}$ In addition to the motor impairments, patients with CP can also show deficits in language development, perception, cognition, and/ or epilepsy. ${ }^{6}$

Movement disorders, namely the impairment of hand motor function, can also be observed in other neurodevelopmental disorders. However, patients with neurodevelopmental disorders that do not primarily affect movement and posture are not considered to have $\mathrm{CP} ;{ }^{7}$ they can generally perform most common movements, but the execution is slower and/or clumsier than in a healthy individual.

Given the impact of impaired motor function on the quality of life, rehabilitation methods that can promote the use of hands in daily activities are very important to patients with hand movement disorders. Several recent studies have highlighted the potential of playing musical instruments in the rehabilitation of hand motor skills in 
stroke patients ${ }^{8-11}$ and in adults with CP. ${ }^{12}$ An acoustic piano provided with keystroke speed sensors has been proposed as a standard tool for the rehabilitation of professional pianists, in order to both improve motor function and assess the outcomes of treatment. ${ }^{13}$ Beneficial effects in the rehabilitation of hand motor function are backed up by improvements in finger movement accuracy, keystroke speed, and timing accuracy. Another recent study in patients with arm paresis caused by stroke showed significant improvements in fine and gross motor dexterities already after 3 weeks of piano lessons. ${ }^{14}$ While experimental evidence supports the employment of piano training in the rehabilitation of hand motor impairments caused by stroke, it remains unclear to what extent it can benefit other clinical populations, in particular patients who, unlike stroke patients, need to learn to execute the movements for the first time (patients with $\mathrm{CP}$ ) or cannot execute regular hand movements accurately (as patients with other neurodevelopmental disorders) and may have concomitant learning difficulties.

Instrumental piano training entails the repetition of fine finger movements as well as finger and hand coordination. Furthermore, the person performing the movement receives an immediate auditory feedback on their performance and can therefore, through it, try to correct their own performance. Playing the piano is likely therefore to promote 1) the development of hand fine motor skills; 2) the integration of audio-visual information with motor control; and 3) the coordination of finger and hand movements. Performing these movements involves the recruitment of different brain areas and functions, ${ }^{15-17}$ and therefore, playing the piano is also likely to promote the communication between different brain areas. Furthermore, piano training has been shown to promote brain plasticity. ${ }^{18,19}$ Hence, while this multisensory character of piano playing may be considered advantageous for therapy, whether it can really facilitate the learning and improvement of hand motor skills in patients with motor disorders of neurodevelopmental origin, who throughout their lives always experienced limited hand motor function and who can have concomitant sensory impairments, remains to be clarified.

The current study addresses this complex question by testing if learning to play the piano can improve finger movement in children and youths with hand motor impairments due to brain injury during development. Eighteen children and youths with impaired hand motor function and similar cognitive abilities received for 1.5 years individual classes of piano with a professional piano teacher. Given the heterogeneous nature of the clinical groups and in order to maximize the probability of a positive outcome, participation in the study was allowed to children with different types of CP and as well as other types of neurodevelopmental disorders. However, because youths with $\mathrm{CP}$ can have contractures or spasticity and these may induce a different evolution of hand skills, training outcome was analyzed, first, separately for the youths with $\mathrm{CP}$ and youths with other neurodevelopmental disorders, and second, over the whole group of participants. The emotional and entertaining character of music entails certainly a motivational factor that was expected to increase the efficacy of the therapy in this young group of patients. Furthermore, the use of piano for the training has the additional advantage that it does not require the child to hold the instrument for a long time, like with the violin, for example.

\section{Methods}

\section{Participants}

Eighteen children and youths (eight male, ten female) showing signs of impaired hand motor function were included in the study. Besides clinical observation, evaluation of the degree of manual skill impairment considered the guidelines of the Manual Ability Classification System for description of manual skills in $\mathrm{CP} .{ }^{20}$ Furthermore, results of the Box-andBlock test ${ }^{21}$ provided further evidence of a reduced manual ability in these children (refer "Results" section). At the start of the study, youths were between 6 and 16 years old. All were recruited from the same day care center for children with motor disabilities. Ten of the children had a diagnosis of infantile CP (named "CP group"). According to the clinical magnetic resonance imaging (MRI) examinations, three patients in this group had visible brain lesions: one presented a large cystic defect in the right temporo-fronto-parietal area with circumjacent gliosis, another showed periventricular leukomalacia consistent with fetal hypoxia, and one presented a large lesion in cortical motor areas (Table 1). Other participants in this group did not show visible brain lesions, or a conclusive examination was not possible, mostly due to movement in the scanner. Eight youths were diagnosed with other development-associated movement disorders, named here as "global retardation and movement coordination disorder (GRMCD) group". (The term used here to name this group is not derived from the literature, and its use is limited to the current paper. This group includes youths with different types of neurodevelopmental disorders). These patients do not present symptoms of spasticity or dyskinesia; they present milder movement disorders, with main symptoms being clumsiness and coordination problems, and they show signs of global 
Table I Participants' clinical data

\begin{tabular}{|c|c|c|c|c|c|c|}
\hline Participant & GMFCS $^{a}$ & $1 Q^{b}$ & $\begin{array}{l}\text { Preferred } \\
\text { hand }\end{array}$ & $\begin{array}{l}\text { Hand with } \\
\text { contracture }\end{array}$ & Diagnosis & MRI examination \\
\hline CPI & 2 & 71 & $\mathrm{~L}$ & NA & Leg-dominated bilateral spastic CP & No examination \\
\hline $\mathrm{CP} 2$ & I & 62 & $\mathrm{~L}$ & NA & Dyskinetic CP & Age-normal image of neurocranium \\
\hline $\mathrm{CP} 3$ & 3 & 81 & $\mathrm{~L}$ & NA & Leg-dominated bilateral spastic CP & Age-normal image of neurocranium \\
\hline $\mathrm{CP} 4$ & 2 & NAss & $\mathrm{R}$ & NA & Leg-dominated bilateral spastic CP & Age-normal image of neurocranium \\
\hline CP5 & I & 71 & $\mathrm{R}$ & L & Unilateral spastic CP & $\begin{array}{l}\text { Large cystic defect on the right } \\
\text { temporo-fronto-parietal area with } \\
\text { discrete circumjacent gliosis, consistent } \\
\text { with ischemia }\end{array}$ \\
\hline CP6 & I & 71 & $\mathrm{~L}$ & $\mathrm{R}$ & Unilateral spastic CP & No examination \\
\hline CP7 & I & 74 & $\mathrm{R}$ & NA & Hypotone CP & Brain parenchyma normal to the age \\
\hline CP8 & I & 92 & $\mathrm{R}$ & L & Unilateral spastic CP & $\begin{array}{l}\text { No report possible due to movement } \\
\text { in the scanner }\end{array}$ \\
\hline CP9 & 2 & 79 & $\mathrm{~L}$ & NA & Leg-dominated bilateral spastic CP & $\begin{array}{l}\text { Periventricular leukomalacia consistent } \\
\text { with fetal hypoxic brain damage }\end{array}$ \\
\hline CPIO & I & NAss & $\mathrm{L}$ & $\mathrm{R}$ & Arm-dominated unilateral spastic CP & $\begin{array}{l}\text { Large lesion in the left hemisphere } \\
\text { covering motor cortical areas }\end{array}$ \\
\hline GRMCDI & 2 & 81 & $\mathrm{R}$ & NA & Global retardation & $\begin{array}{l}\text { No indication of intracranial expansion, } \\
\text { ischemia, or bleeding }\end{array}$ \\
\hline GRMCD2 & 1 & 79 & $\mathrm{R}$ & NA & Developmental retardation & $\begin{array}{l}\text { Asymmetry of the posterior horn. } \\
\text { Myelination defect }\end{array}$ \\
\hline GRMCD3 & 1 & 101 & $\mathrm{~L}$ & NA & Neurofibromatosis & $\begin{array}{l}\text { Large pineal gland cyst; no compression } \\
\text { of neighboring structures. Lesion in } \\
\text { the front part of the midbrain and in } \\
\text { Globus Pallidus, both sides }\end{array}$ \\
\hline GRMCD4 & I & 71 & $\mathrm{R}$ & NA & Global retardation & Age-normal image of neurocranium \\
\hline GRMCD5 & I & 94 & $\mathrm{~L}$ & NA & Global retardation & Age-normal image of neurocranium \\
\hline GRMCD6 & 1 & 58 & $\mathrm{R}$ & NA & Global retardation & $\begin{array}{l}\text { No indication of expansion or bleeding, } \\
\text { but detailed examination not possible } \\
\text { due to movement in the scanner }\end{array}$ \\
\hline GRMCD7 & 1 & 81 & $\mathrm{R}$ & NA & Fragile $X$ syndrome & Age-normal image of neurocranium \\
\hline GRMCD8 & I & 79 & $\mathrm{R}$ & NA & Global retardation & No examination \\
\hline
\end{tabular}

Notes: a Calculated according to the GMFCS. ${ }^{22}$ blQ is calculated according to the German version of the Wechsler Intelligence Scale, Hamburg Wechsler Intelligence Scale for Children (HAWIK-IV). ${ }^{23}$

Abbreviations: GMFCS, Gross Motor Function Classification System; IQ, intelligence quotient; MRI, magnetic resonance imaging; CP, cerebral palsy; GRMCD, global retardation and movement coordination disorder; L, left; R, right; NA, not applicable; NAss, not assessed.

retardation. Two children in this group showed visible brain lesions: one presented an asymmetry of the posterior horn and a myelination defect; another presented a large pineal cyst and lesions in the front part of the midbrain and in Globus Pallidus. The other participants in this GRMCD group presented no visible lesions, or one was not examined.

Table 1 gives an overview of the clinical data collected for all participants: Gross Motor Function Classification System (GMFCS) level, ${ }^{22}$ handedness, intelligence quotient level, ${ }^{23}$ clinical diagnosis, and indication of detectable brain lesions with MRI.

Inclusion in the study required the participant to 1) have impaired hand motor function but being able nevertheless to do some movements with the hands, 2) never have had piano classes before, and 3) be able to pay attention to given instructions and to concentrate in a task. The requirement of being able to make some movements with the hands implied the exclusion of children with severe spasticity and/or contractures in both hands.

Children and parents were directly contacted and made aware of the study. Participation was voluntary, and the parents' consent was obtained before starting the study. All procedures in the study were approved before commencement by the Ethics Committee of the Faculty of Medicine of the Technische Universität München.

All children continued their regular therapy program (physiotherapy twice a week and swimming therapy once a week) during the study.

\section{Piano training}

All children and youths had 30-45 minutes of piano training with a professional piano teacher twice a week for 18 months. 
Both piano teachers involved in the study had experience in working with disabled persons. An 88-key Musical Instrument Digital Interface (MIDI) controller keyboard $\left(\right.$ Casio $\left.^{\circledR}\right)$ was used in the training sessions and to run some of the motor skills tests (refer "Piano tests" section). Every training session started with preliminary finger movements for warming up the hands. The exercises were as follows: a shortened pentatonic scale moving up and down was played with the right hand, beginning with the thumb and ending with the little finger. The exercise was then repeated with the left hand and then with both hands at the same time. By following the sequence one finger after the other, the selective motor activation of individual fingers could be qualitatively estimated by the music teacher. After these exercises, small pieces of Russian piano music and some popular songs were learned. Sometimes, four-hand piano pieces (eg, dances) were played by the teacher and the pupil or even by two pupils. The patients also took part in a concert at the end of the study where they played four-hand piano pieces together with healthy children of similar age.

\section{Experimental tests of motor function}

All tests and assessments were carried out at the daycare center.

\section{Piano tests}

The same 88-key MIDI piano employed in the piano classes was used to measure the kinematics and dynamics of keystroking and therefore the evolution of specific finger motor function during the training. For this purpose, special piano exercises were conceptualized by the researchers and piano teachers. The MIDI piano was connected to a computer where the precise timing, strength of keystrokes, and the pressed piano keys were recorded. Assessment and graphical representation of the data collected were done with specialized software developed together by the researchers and piano teachers. Three special piano tests were designed to evaluate changes in fine finger motor function. The tests, described below, were conducted every 3 months. The exercises were video-recorded to be able to exclude aberrant data caused by, for example, wrong scale on the piano, an unfocused child, or an incorrect sitting position.

\section{Test I}

Every finger of the nondominant hand, beginning with the thumb, then index, middle finger, ring, and finally the little finger, struck eight times consecutively the same piano key. To help the child develop a steady finger-tapping movement, a rhythmic song/poem was read out loud by the piano teacher and also by the pupil, while the latter was playing. During the singing, the different fingers are "called"/mentioned to help the child use them successively and in an active way (by "calling" them himself/herself). The method uses standard teaching practices in music classes and was particularly useful for pupils who experienced more difficulties selecting and using different fingers separately. For each child, the mean time interval between two consecutive keystrokes and its associated standard deviation were computed for both hands separately. The latter reflects the variability of successive keystrokes (by different fingers) and therefore provides a measure of how uniform the finger movements are.

\section{Test 2}

The same as test 1 but done with the dominant hand.

\section{Test 3}

The same as test 1 but done with the same fingers of both hands simultaneously. The mean time interval between keystrokes of the same two fingers in the two hands and the associated standard deviation were computed. The latter provides an indication of how variable the simultaneous keystroking with the two hands is. That is, it provides an indication of how coordinated the movement between the same fingers of the two hands is.

These measures were collected before, during, and at the end of the piano training.

\section{Box-and-Block test}

The Box-and-Block test ${ }^{21}$ was employed in the assessment of fine and gross motor dexterity before and after the piano training. Children sat in front of a table and were instructed to lift and move wooden cubes $2.5 \mathrm{~cm} \times 2.5 \mathrm{~cm} \times 2.5 \mathrm{~cm}$ in size from one side of the table to the other over a $15 \mathrm{~cm}$ high partition that was in the middle. They were asked to move as many cubes over the partition as possible within 1 minute. The total number of cubes moved by each hand separately was counted and compared to reference values according to the children's age. ${ }^{21,24}$

\section{Hand dynamometer test}

A commercial hand dynamometer (Baseline ${ }^{\circledR}$ ) was used to measure the hand force before and after the piano training. The device is easy to use providing immediate information about the strength of the hand simply by clenching the device. The dominant hand is typically the stronger hand (larger force). 


\section{Statistical analysis}

Statistical evaluation of experimental data used functions available in the MATLABC R2014b Statistical Toolbox. Repeated-measures analysis of variances (ANOVAs) tested effects of training (by testing effects of session - "before" vs "after" the training), group ("CP" vs "GRMCD"), and age, as well as age $\times$ group interaction effects. The age factor was included as this could correlate with children's motor ability, as measured by the experimental tests, and to account for potential differences between the two groups. As shown in the "Results" section, this was not the case, and the influence of hand motor ability before starting the training was tested instead.

\section{Results \\ Piano tests}

Figure 1 shows the results of the piano tests. Figure $1 \mathrm{~A}$ and $\mathrm{B}$ shows the average time interval between consecutive strokes with the same finger at different measurement sessions relative to the average time interval registered individually in the first session. The data provide therefore a measure of the development relative to the first session. Because it is a ratio, it has no units and varies by approximately 1 . This provides a useful measure of individual performance across sessions, and given the large variability in manual ability across participants, also a measure comparable to other participants. Figure $1 \mathrm{C}$ and $\mathrm{D}$ shows the standard deviation associated to the time interval between consecutive strokes with the same finger divided by the average time interval, in percentage. Lower values indicate more regular keystroking, and hence better fine motor control. Ratios were calculated separately for each individual and then averaged across participants.

For both CP (Figure 1A) and GRMCD (Figure 1B) groups, the average time interval between consecutive strokes remained practically unchanged throughout the training period. However, mean deviations decreased with session for both groups (Figure 1C and D).

A repeated-measures model was fitted to the standard deviation of stroke interval (in milliseconds) collected for all participants, both for the dominant and nondominant hands. The model included two between-subject factors ("group" [CP and GRMCD] and "age"), an interaction factor (group $\times$ age), and two within-subject factors ("hand" ["dominant" and "nondominant"] and "session" [one to six measurement sessions]). Mauchly's test indicated violation of the assumption of sphericity, and therefore, the Greenhouse-Geisser correction of sphericity was applied $(\varepsilon=0.27) .{ }^{25}$ Results showed a significant effect of session $(F[11,44]=3.97, P<0.05)$ but no significant effect of group $(F[11,44]=1.06, P=0.40)$ or age
$(F[11,44]=2.33, P=0.13)$. Also, the interaction group $\times$ age was not significant $(F[11,44]=1.00, P=0.42)$.

A similar model was also fitted to the mean stroke interval, and the degrees of freedom corrected using the Greenhouse-Geisser estimates of sphericity $(\varepsilon=0.21)$. Statistical tests showed no significant effect of session $(F[11,44]=0.49, P=0.65)$, group $(F[11,44]=0.46, P=0.57)$, age $(F[11,44]=0.40, P=0.71)$, and the interaction group $\times$ age $(F[11,44]=0.44, P=0.69)$ on the mean stroke interval.

In addition to effects of age and group, the effect of the initial score of the participant in the piano test on the final change at the end of the training, as assessed with the piano tests, was sought with a simple regression analysis. In order to boost the power of the analysis, this was performed without distinguishing groups or hands tested, that is, the sample included data from all participants and all hands tested. This provided an additional measure of the potential benefit of piano training, irrespective of the patient group or hand. The results of the regression analysis are presented in Figure 1E and F: the difference in mean interval between the first and the last test session was correlated with the mean stroke interval in the first session (ie, [mean interval in last session] - [mean interval in first session]) (Figure 1E) and the difference in deviation ([mean deviation in last session] - [mean deviation in first session]) with the mean deviation recorded in the first session (Figure 1F). A significant $(P<0.05)$ correlation was found both for the difference in mean interval (Figure $\left.1 \mathrm{E} ; F(1,28)=16.88, P<0.01, R^{2}=0.38\right)$ and for the difference in mean interval deviation (Figure 1F; $F[1,28]=184.63$, $P<0.01, R^{2}=0.87$ ), meaning that, for this group of participants, the initially (before training) recorded mean interval and deviation between consecutive finger strokes explained $38 \%$ and $87 \%$ of the variance observed in the final change registered for those variables, respectively.

\section{Box-and-Block test}

For all participants, the number of cubes moved with any of the hands was below the average number of cubes reported to be moved by their healthy peers of similar age, ${ }^{21}$ consistent with the initial diagnosis of impaired hand motor function. Only eight out of 30 "tested hands" in total (tests missing for three patients for the first test session) were within the range of blocks observed in children of similar age group (for comparison, we used Table 2 in Ref 21).

The average results of the Box-and-Block test before and after piano training showed an improvement of 5.1 and 3.4 blocks for the nondominant and dominant hands, respectively, for the CP group (Figure 2A). The large standard 

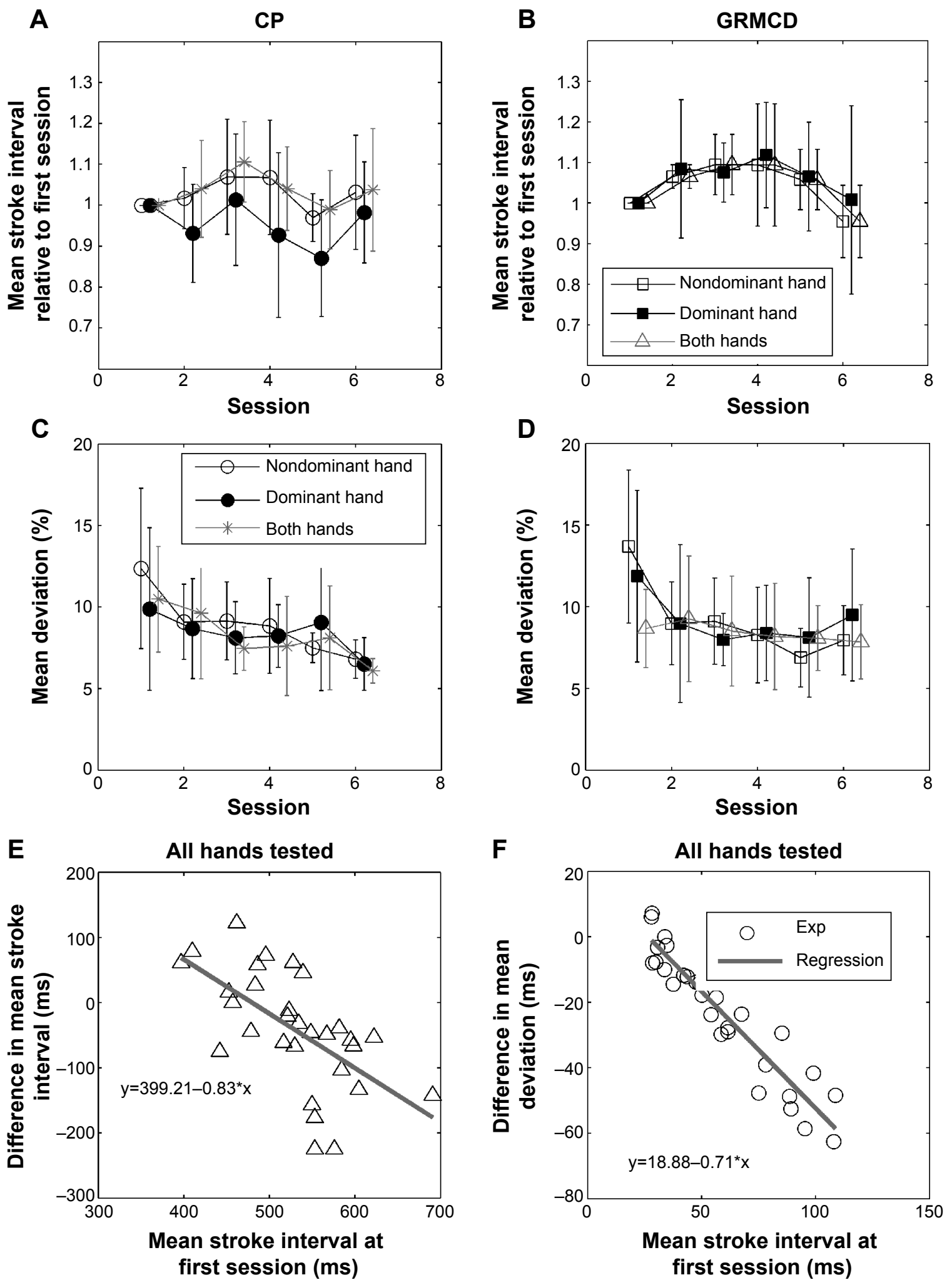

Figure I Results of the piano tests.

Notes: (A) Mean stroke time and associated standard deviation, calculated across participants in the CP group, as a function of measurement session (one to six) with the dominant hand (black circles), nondominant hand (open circles), and both hands simultaneously (gray asterisks and line). Mean stroke times are plotted relative to the value in the first session. (B) The same as (A) but for the GRMCD group, for the dominant (black squares), nondominant (open squares), and both (gray triangles) hands. (C) Mean, across participants in the CP group, of the ratio, standard deviation associated to mean stroke time/mean stroke time, in percentage, as a function of session. (D) The same as (C) but for the GRMCD group. (E) Difference ([mean stroke interval in last session] - [mean stroke interval in first session]) as a function of the mean stroke interval in the first session. Results are presented for all participants and hands tested (experimental [Exp] data illustrated with open triangles). The gray line illustrates the linear regression obtained for this data sample, with the corresponding line equation indicated on the side. (F) Difference ([mean deviation in last session] - [mean deviation in first session]) as a function of the deviation recorded in the first session, for all participants and hands tested (Exp data illustrated by open circles).

Abbreviations: $\mathrm{CP}$, cerebral palsy; GRMCD, global retardation and movement coordination disorder. 

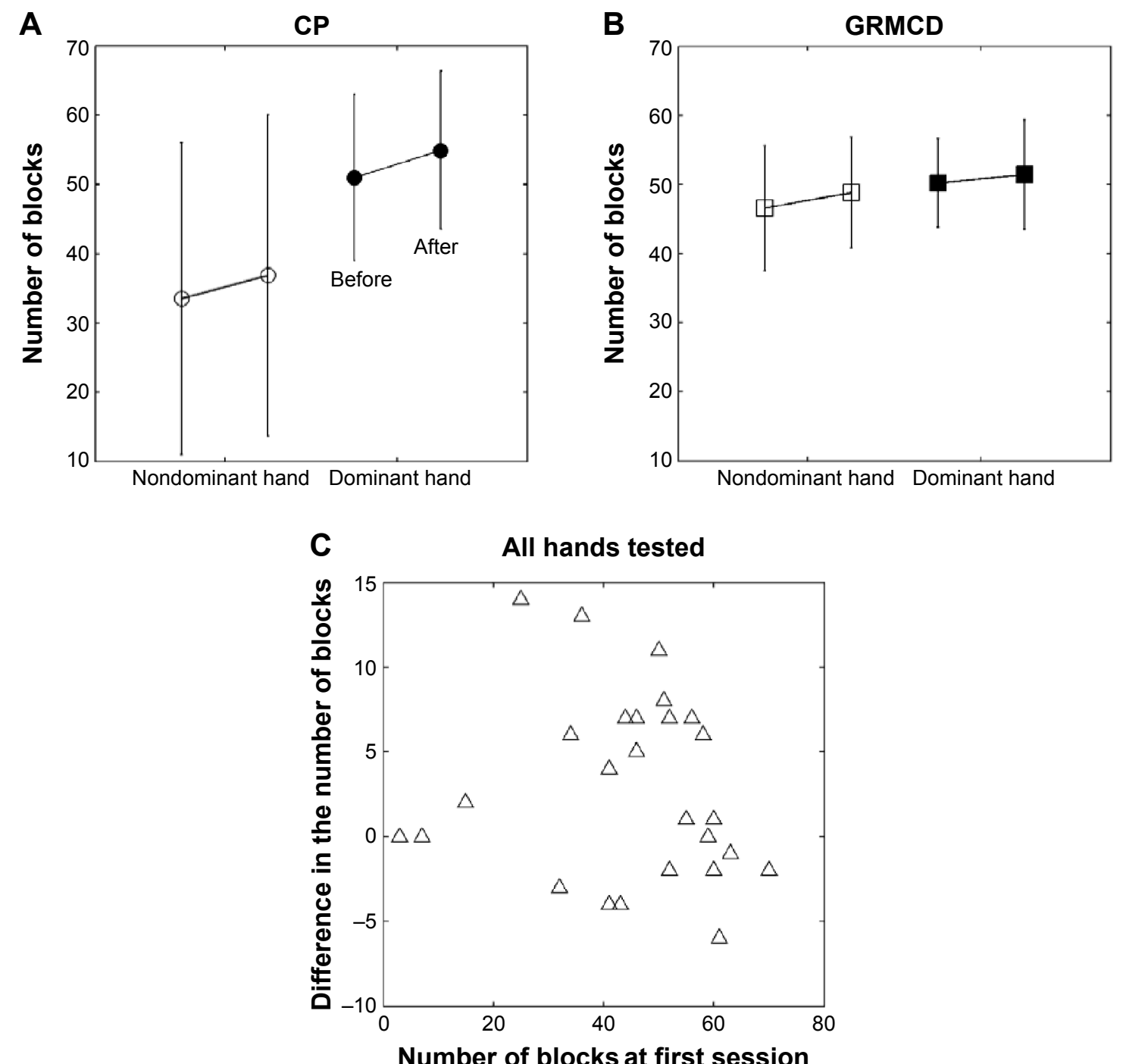

Figure 2 Results of the Box-and-Block test.

Notes: (A) Average number of blocks moved before (left black circle and left open circle) and after (right black circle and right open circle) the training, obtained separately with the dominant (filled circles) and nondominant (open circles) hands, for the CP group. (B) The same as (A) but for the GRMCD group. (C) Difference (number of blocks after the training - number of blocks before the training), as a function of the number of blocks moved before the training, in the first session. Data are presented for all hands tested (irrespective of group).

Abbreviations: CP, cerebral palsy; GRMCD, global retardation and movement coordination disorder.

deviation especially noticeable with the nondominant hand in the CP group reflect the large difference in performance between patients with unilateral $\mathrm{CP}$ and patients with bilateral CP. Changes were smaller for the GRMCD group: 0.94 and -0.17 blocks on average for the nondominant and dominant hands, respectively (Figure 2B). A two-way repeated-measures ANOVA on the results obtained with the nondominant hand revealed no statistical significant effect of session $(F[1,11]=3.61, P=0.084)$ or of group $(F[1,11]=1.19$, $P=0.2992$ ). Furthermore, no significant interaction between the two factors was found $(F[1,11]=0.14, P=0.7174)$. No significant changes were obtained with the dominant hand either (effect of session: $F[1,11]=3.1, P=0.106$; effect of group: $F[1,11]=0.12$, $P=0.7335$; interaction: $F[1,11]=0.65, P=0.4378)$.
As with the piano tests, also here, changes in the results of the Box-and-Block test, before and after the piano training, were analyzed as a function of the test outcome in the first session, to assess the extent to which improvements depended on the initial "motor ability" of the participants. The analysis however did not show a significant effect of the initial score in the test on the change recorded after the piano training had ended (Figure 2C).

\section{Hand dynamometer test}

The mean values of maximum grip force for participants in the $\mathrm{CP}$ and GRMCD groups before and after the piano training are shown in Figure $3 \mathrm{~A}$ and B, respectively. No significant changes in grip strength were registered for the nondominant hand (two-way 

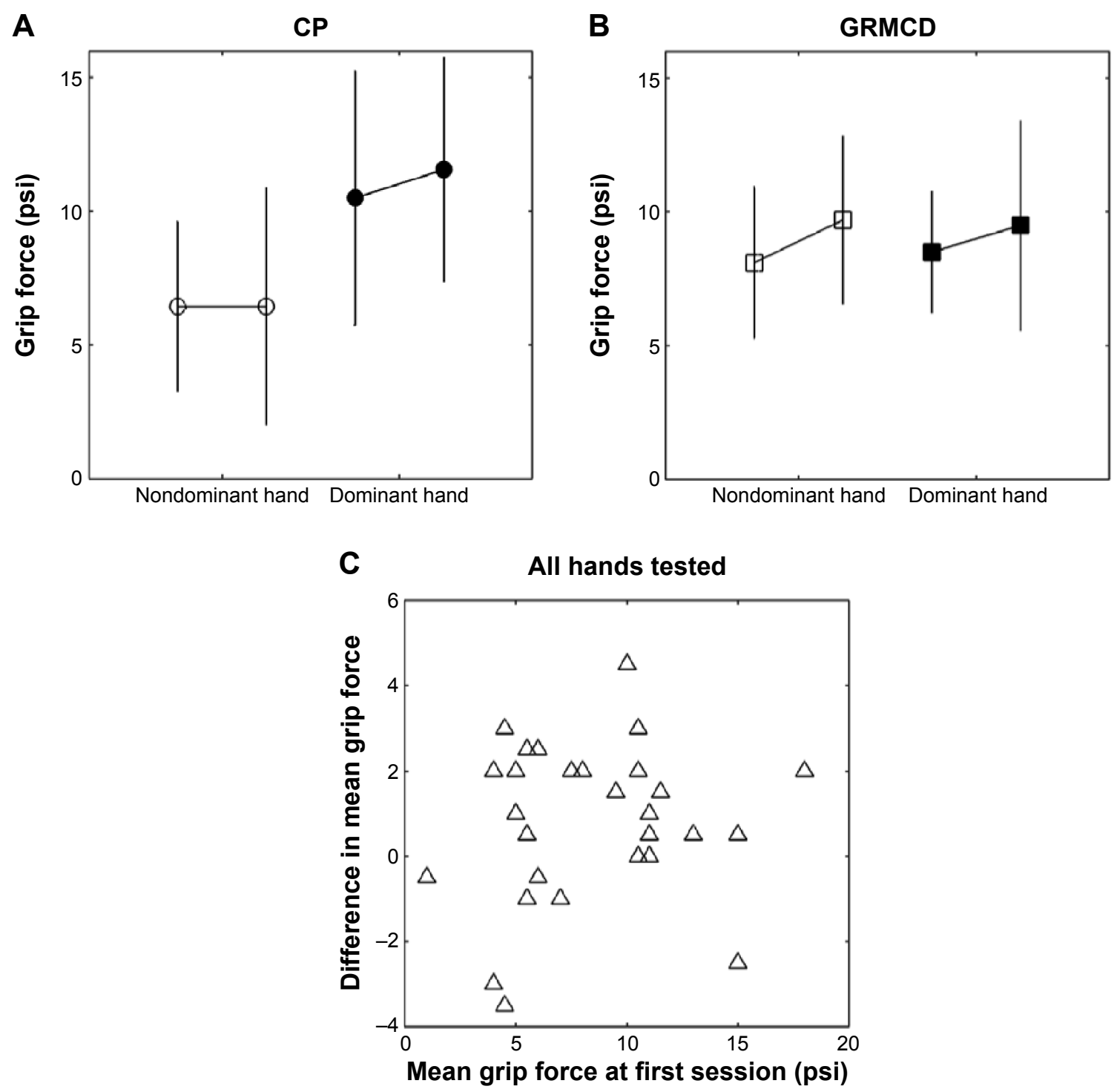

Figure 3 Results of the grip force test.

Notes: (A) Average maximum grip force (in psi) and associated standard deviation measured before (left open circle and left black circle) and after (right open circle and right black circle) the piano training with the dominant (black filled circles) and nondominant (open circles) hands, respectively, for the participants in the CP group. (B) The same as (A) but for the GRMCD group. (C) Difference (mean grip force after the piano training - mean grip force before the training) as a function of the grip force measured in the first experimental session. Data are presented for all hands tested.

Abbreviations: CP, cerebral palsy; GRMCD, global retardation and movement coordination disorder.

repeated-measures ANOVA - effect of session: $F[1,12]=1.28$, $P=0.2792$; effect of group: $F[1,12]=1.39, P=0.2605$ ) or for the dominant hand (effect of session: $F[1,12]=4.32, P=0.0598$; effect of group: $F[1,12]=0.72, P=0.4124)$.

A regression analysis testing the effect of the initial hand grip force on the difference in grip force registered at the end of the piano training (Figure 3C) yielded no significant results.

\section{Discussion}

The regularity between consecutive finger strokes during rhythmic piano exercises improved after one and a half years of intensive piano training, in a group of youths with impaired hand motor function resulting from damage to the developing brain. Although eight out of the 16 youths had a diagnosis of $\mathrm{CP}$, experimental tests did not yield differences in relation to the group of youths with milder hand motor impairment, associated to other neurodevelopmental disorders. Most of the variability in mean interval and interval deviation (ie, increase in regularity), as measured by the piano tests, was accounted for by the initial ability of the participant, with larger improvements (ie, stronger reductions in finger stroke interval and interval deviations) recorded for those patients who showed more irregular finger movements at the start of the study. 
It can rightly be argued that the effects seen are not due to piano training but instead reflect an expected manual skill development that takes place within normal physical development. To rule this factor out, a control group composed of children with similar clinical signs and of similar age but who have not received the piano training should have been considered in the study. Therefore, the results presented are not conclusive, and further tests remain necessary to confirm the association between the improvements observed and the piano training.

One observation however let us envisage that changes observed can be associated to the piano training: the deviation of the keystroke interval decreased more steeply between the first measurement sessions (namely between the first and second session) than between the following sessions, that is, there was a faster change in the beginning of the training than toward the end. Had the changes resulted from normal manual skill development, the evolution would have likely been more gradual and uniform throughout time.

The heterogeneous origin of motor impairments across participants in both CP and GRMCD groups also introduced variability in the results and consequently a reduction in power of the effects of training. The original aim of the study was to test if children/youths with impaired hand motor skills due to early brain damage could benefit from intensive and prolonged piano training, independent of the specific cause of the impairment. Furthermore, $\mathrm{CP}$ is, per definition, an umbrella term covering a diverse group of motor impairments. ${ }^{26}$ Despite the variety of underlying causes and associated brain lesions that are seen in the experimental group, testing a more diverse group of patients rather than testing only patients with a specific type of damage was intended to increase the probability of having a positive outcome in the training. Variable results were observed at the individual level, and also, no differences were observed between $\mathrm{CP}$ and GRMCD groups. The low number of participants further added to the lack of larger experimental effects. A statistical power analysis performed for sample size estimation and based on the piano results presented here and testing for a significant correlation between the reduction in stroke interval with the training and the stroke interval data collected at the start of the study (Figure 1E) yielded, for an alpha $=0.05$ and power $=0.8$, a projected sample size of 16 (GPower 3.1.9.2 software ${ }^{27}$ ). This calculation is based here on data collected for all hands tested from all participants. To analyze the outcome for dominant and affected hands separately, at least 16 data points would be required for each hand side.

The improvements here reported are in agreement with similar studies on instrumental training in other clinical populations, namely stroke patients ${ }^{8}$ and in adults with
$\mathrm{CP},{ }^{12}$ and support the use of piano training in the rehabilitation of youths with hand motor impairments due to damage to the developing brain. The use of musical keyboards by adults with CP has also been supported by improvements in manual dexterity and finger strength after $6-9$ weeks of piano training. ${ }^{12}$

A parallel study in our laboratory reported enhanced connectivity between the contralateral primary motor cortex and the cerebellum ipsilateral to the dominant hand in a group of youths with $\mathrm{CP}$ after receiving piano training for 18 months. ${ }^{28}$ Although some of the children participated both in the imaging and in the current study, the experimental populations were however different between the two studies. It is possible that the changes in finger mobility observed in the current study in the form of more uniform finger strokes are the behavioral counterpart of the increased connectivity reported after the functional MRI assessment.

The intensive piano training promoted the motor learning of specific manual skills that were captured here by the piano tests. In comparison to other motor training therapies, which also promote motor learning through repetition of movements, piano training brings motor execution and auditory perception together, this way promoting the interaction of different brain areas. Also, the simultaneous acoustic and sensory-motor feedback during playing helps to switch the attentional focus in these patients from the execution of difficult hand/finger movements to music and to emotional experience. This clearly increased motivation in the youths, as reported by the music teachers. Additional psychological assessments of the effects of piano training would have helped to clarify the additional benefits that piano training can bring to this clinical group.

The effects of piano training produced changes in piano test results. No changes were detected by the Box-and-Block or by the hand grip force test. How the effects of piano training transfer to other manual abilities remains hence to be clarified. Nevertheless, in face of the changes here detected, in terms of reduction in mean interval deviation, effects of piano training are likely to induce improvements in manual tasks requiring good hand dexterity more than hand force. The physical contact with a real piano keyboard, together with the auditory feedback, may help further to gain sensitivity for the fingers and finger movement. In a study with professional pianists, the timing accuracy of finger movements in playing the piano was enhanced by the tactile information transmitted during the contact of the fingers with the keys. ${ }^{29}$

Overall, the effects observed are encouraging, but as pointed above, studies remain necessary to confirm the beneficial effects here observed and especially to investigate the benefits of piano 
training in more homogeneous groups. Furthermore, given that visible and measurable effects are more likely to occur in the beginning of the training and in a relatively short period of time,${ }^{30}$ it may be advisable to plan shorter periods of training, and in this way, to also give the chance to control participants who initially were not given the training to have it afterward, in a repeated-measures design with crossover. The shorter training and assessment will also reduce the influence of growth on the development of finger and manual mobility.

\section{Acknowledgment}

We thank the Buhl-Strohmaier Foundation for the financial support to conduct this study.

\section{Disclosure}

The authors report no conflicts of interest in this work.

\section{References}

1. Odding E, Roebroeck ME, Stam HJ. The epidemiology of cerebral palsy: incidence, impairments and risk factors. Disabil Rehabil. 2006;28(4): 183-191.

2. Debert C, Herter T, Scott S, Dukelow S. Robotic assessment of sensorimotor deficits after traumatic brain injury. J Neurol Phys Ther. 2012;36(2):58-67.

3. Lawrence D, Kuypers $H$. The functional organisation of the motor system in the monkey: I. The effects of bilateral pyramidal lesions. Brain. 1967;91:1-14.

4. Staudt M, Gerloff C, Grodd W, Holthausen H, Niemann G, KrägelohMann I. Reorganization in congenital hemiparesis acquired at different gestational ages. Ann Neurol. 2004;56(6):854-863.

5. Gordon AM, Bleyenheuft Y, Steenbergen B. Pathophysiology of impaired hand function in children with unilateral cerebral palsy. Dev Med Child Neurol. 2013;55(s4):32-37.

6. Stotz S. Treatment of Cerebral Palsy. Munich: Pflaum Verlag; 2000.

7. Rosenbaum P, Paneth N, Leviton A, Goldstein M, Bax M. A report: the definition and classification of cerebral palsy April 2006. Dev Med Child Neurol Suppl. 2007;109:8-14.

8. Schneider S, Schönle PW, Altenmüller E, Münte TF. Using musical instruments to improve motor skill recovery following a stroke. J Neurol. 2007;254(10):1339-1346.

9. Altenmüller E, Marco-Pallares J, Münte T, Schneider S. Neural reorganization underlies improvement in stroke - induced motor dysfunction by music - supported therapy. Ann N Y Acad Sci. 2009;1169(1): 395-405.

10. Schneider S, Münte T, Rodriguez-Fornells A, Sailer M, Altenmüller E. Music-supported training is more efficient than functional motor training for recovery of fine motor skills in stroke patients. Music Percept. 2010;27:271-280.

Neuropsychiatric Disease and Treatment

\section{Publish your work in this journal}

Neuropsychiatric Disease and Treatment is an international, peerreviewed journal of clinical therapeutics and pharmacology focusing on concise rapid reporting of clinical or pre-clinical studies on a range of neuropsychiatric and neurological disorders. This journal is indexed on PubMed Central, the 'PsycINFO' database and CAS,
11. Grau-Sánchez J, Amengual JL, Rojo N, et al. Plasticity in the sensorimotor cortex induced by Music-supported therapy in stroke patients: a TMS study. Front Hum Neurosci. 2013;7:494.

12. Chong HJ, Cho SR, Jeong E, Kim SJ. Finger exercise with keyboard playing in adults with cerebral palsy: a preliminary study. $J$ Exerc Rehabil. 2013;9(4):420-425.

13. Minetti AE, Ardigo LP, McKee T. Keystroke dynamics and timing: accuracy, precision and difference between hands in pianist's performance. J Biomech. 2007;40(16):3738-3743.

14. Villeneuve M, Lamontagne A. Playing piano can improve upper extremity function after stroke: case studies. Stroke Res Treat. 2013;2013: 159105.

15. Bangert M, Peschel T, Schlaug G, et al. Shared networks for auditory and motor processing in professional pianists: evidence from fMRI conjunction. Neuroimage. 2006;30(3):917-926.

16. Baumann S, Koeneke S, Schmidt C, Meyer M, Lutz K, Jancke L. A network for audio-motor coordination in skilled pianists and nonmusicians. Brain Res. 2007;1161:65-78.

17. Bengtsson S, Nagy Z, Skare S, Forsman L, Forssberg H, Ullén F. Extensive piano practicing has regionally specific effects on white matter development. Nat Neurosci. 2005;8:1148-1150.

18. Zatorre R, Chen J, Penhune V. When the brain plays music: auditorymotor interactions in music perception and production. Nat Rev. 2007;8: 547-558.

19. Herholz S, Zatorre R. Musical Training as a framework for brain plasticity: behavior, function, and structure. Neuron. 2012;76(3):486-502.

20. Eliasson AC, Krumlinde-Sundholm L, Rösblad B, et al. The manual ability classification system (MACS) for children with cerebral palsy: scale development and evidence of validity and reliability. Dev Med Child Neurol. 2006;48(07):549-554.

21. Mathiowetz V, Federman S, Wiemer D. Box and Block test of manual dexterity: norms for 6-19 year olds. CJOT. 1985;52(5):241-245.

22. Rosenbaum PL, Palisano RJ, Bartlett DJ, Galuppi BE, Russell DJ. Development of the gross motor function classification system for cerebral palsy. Dev Med Child Neurol. 2008;50(4):249-253.

23. Petermann F, Petermann U. HAWIK-IV. Bern: Huber; 2010.

24. Mathiowetz V, Wiemer D, Federman S. Grip and pinch strength: norms for 6-to 19-year-olds. Am J Occup Ther. 1986;40(10):705-711.

25. Field A. Discovering Statistics using SPSS. Los Angeles: Sage; 2009.

26. Aisen ML, Kerkovich D, Mast J, et al. Cerebral Palsy: clinical care and neurological rehabilitation. Lancet. 2011;10:844-852.

27. Faul F, Erdfelder E, ALang A-G, Buchner A. G*Power 3: A flexible statistical power analysis program for the social, behavioral and biomedical sciences. Behv Res Methods. 2007;39:175-191.

28. Alves-Pinto A, Turova V, Blumenstein T, Thienel A, Lampe R. fMRI assessment of neuroplasticity in youths with neurodevelopmentalassociated motor disorders after piano training. Eur J Paediatr Neurol. 2015;19(1):12-15.

29. Goebl W, Palmer C. Tactile feedback and timing accuracy in piano performance. Exp Brain Res. 2008;186(3):471-479.

30. Lahav A, Saltzman E, Schlaug G. Action representation of sound: audiomotor recognition network while listening to newly acquired actions. J Neurosci. 2007;27(2):308-314.

and is the official journal of The International Neuropsychiatric Association (INA). The manuscript management system is completely online and includes a very quick and fair peer-review system, which is all easy to use. Visit http://www.dovepress.com/testimonials.php to read real quotes from published authors. 